\title{
Effects of Stimulation Mode, Level and Location on Forward-Masked Excitation Patterns in Cochlear Implant Patients*
}

\author{
Monita Chatterjee, John J. Galvin III, Qian-Jie Fu, and Robert V. Shannon \\ Department of Auditory Implants and Perception, House Ear Institute, 2100 W. Third St., Los Angeles, CA 90057, USA
}

Received: 13 July 2005; Online publication: 4 November 2005

\begin{abstract}
In multi-channel cochlear implants, electrical current is delivered to appropriate electrodes in the cochlea to approximate the spatial representation of speech. Theoretically, electrode configurations that restrict the current spread within the cochlea (e.g., bi- or tripolar stimulation) may provide better spatial selectivity, and in turn, better speech recognition than configurations that produce a broader current spread (e.g., monopolar stimulation). However, the effects of electrode configuration on supra-threshold excitation patterns have not been systematically studied in cochlear implant patients. In the present study, forward-masked excitation patterns were measured in cochlear implant patients as functions of stimulation mode, level and location within the cochlea. All stimuli were 500 pulses-per-second biphasic pulse trains $(200 \mu \mathrm{s} /$ phase, $20 \mu$ inter-phase gap). Masker stimuli were $200 \mathrm{~ms}$ in duration; the bi-polar configuration was varied from narrow $(\mathrm{BP}+1)$ to wide $(\mathrm{BP}+17)$, depending on the test condition. Probe stimuli were $20 \mathrm{~ms}$ in duration and the maskerprobe delay was $5 \mathrm{~ms}$; the probe configuration was fixed at $\mathrm{BP}+1$. The results indicated that as the distance between the active and return electrodes in a bi-polar pair was increased, the excitation pattern broadened within the cochlea. When the distance

\footnotetext{
* Portions of this work were presented at the 12th International Symposium of Hearing (2001) held at Mierlo, The Netherlands. Present address: Monita Chatterjee, Department of Hearing and Speech Sciences, University of Maryland, 0100 LeFrak Hall, College Park, MD 20742, USA.

Correspondence to: Monita Chatterjee - Department of Hearing and Speech Sciences · University of Maryland - 0100 LeFrak Hall, College Park, MD 20742, USA. Telephone: +1 301405 7716; fax: +1 301314 2023; email: mchatterjee@hesp.umd.edu
}

between active and return electrodes was sufficiently wide, two peaks were often observed in the excitation pattern, comparable to non-overlapping electric fields produced by widely separated dipoles. Analyses of the normalized data showed little effect of stimulation level on the shape of the excitation pattern.

\section{INTRODUCTION}

The shape and extent of the excitation pattern in the cochlea is expected to play an important role in auditory perception. With cochlear implants (CIs), this pattern may be influenced by a number of factors, including the shape/extent of the stimulating electric field, the density of surviving auditory neurons, the proximity between electrodes and neural tissue, the location of the implanted electrodes within the cochlea, etc. These factors may vary widely across cochlear implant patients, and may contribute to the considerable variability observed in patient outcomes.

In CIs, the electrode configuration (or, stimulation mode) is used to manipulate the extent of the current delivered within the cochlea. Present day CI speech processors commonly use bi-polar or monopolar electrode configurations. With bi-polar configurations, current flows between an active and return electrode within the cochlea; with monopolar configurations, current flows between an active electrode within the cochlea and an extracochlear return electrode. For a fixed loudness level, bi-polar stimulation theoretically produces a more focused spread of excitation within the cochlea than does monopolar stimulation: the smaller the distance between the active and return electrodes, the less spread of current 
through the cochlea, which may provide better spatial selectivity and presumably better speech recognition. Recently, tri-polar configurations have been shown to further restrict the excitation in the cochlea (Bierer and Middlebrooks 2002, 2004; Snyder et al. 2004). In present-day CI speech processing, monopolar stimulation is more commonly used than bi-polar stimulation because thresholds and comfortable listening levels generally require much less current with monpolar than with bi-polar stimulation. The broader spread of excitation with monopolar stimulation has been thought to provide less spatial selectivity, and presumably poorer speech recognition. However, speech recognition with monopolar stimulation has been shown to be equal to and even better than that with bi-polar stimulation (Pfingst et al. 1997). Performance for a variety of speech perception and psychophysical tasks (e.g., intensity, rate discrimination) does not seem depend strongly on stimulation mode, as long as the relative loudness is comparable (Morris and Pfingst 2000; Franck et al. 2003). In a study comparing effects of narrow bi-polar, broad bipolar and monopolar stimulation, Pfingst et al. (2001) found that the location of stimulation, rather than electrode configuration, was the dominant factor in CI subjects' speech recognition scores. Boex et al. (2003) recently measured forward masking patterns for different CI devices and electrode configurations and found no significant difference between in the amount of masking produced by monopolar or bipolar stimulation, in two CI devices. However, they did find a significant negative correlation between the amount of masking estimated at a distance of 4 $\mathrm{mm}$ from the masker electrode (a measure which is also related to the width of the forward masking pattern) and consonant recognition scores.

Recent physiological studies have explored the effects of electrode configuration and channel interaction. Bierer and Middlebrooks (2002) measured neural spike activity along the tonotopic axis of the primary auditory cortex in guinea pigs for singlepulse stimuli in monopolar, bi-polar or tri-polar stimulation modes; tri-polar stimulation produced the most 'spatially compact' excitation, followed by bi-polar, then monopolar stimulation. Snyder et al. (2004) found similar effects of electrode configuration on neural activation patterns in the inferior colliculus of the guinea pig, for single-channel stimuli.

Changes in the excitation pattern produced by varying CI stimulation parameters (e.g., electrode configuration, electrode location, stimulation level, etc.) can be measured at supra-threshold levels using a forward masking technique, in which forward-masked thresholds are compared to unmasked thresholds across the electrode array. The peak threshold shift will generally be found in the region/s of the cochlea that respond most vigorously to the masker; threshold shifts will be lower in regions less responsive to the masker. For short masker-probe inter-stimulus delays, the spatial pattern of forward-masked threshold shifts is thought to reflect the neural excitation pattern evoked by the masker. Several investigators have used forward masking to measure spatial excitation patterns in CI patients (Shannon 1983; Tong and Clark 1986; Lim et al. 1989; Chatterjee and Shannon 1998; Throckmorton and Collins 1999; Nelson and Donaldson 2002; Boex et al. 2003). Chatterjee and Shannon (1998) measured forward masking patterns in CI patients; they found good spatial selectivity for narrowly configured electrode pairs, and that the excitation patterns changed with masker level in a fairly linear fashion. In general, these studies have shown that masking is greatest in the vicinity of the masker, and decays as the masker and probe become more spatially remote (at a rate that varies according to stimulus, electrode location and subject). In most of these previous studies, narrow bi-polar electrode configurations were used for maskers and probes. Thus while the effect of electrode location and stimulation level has been explored in previous studies, the effect of electrode configurations for fixed electrode locations has not been fully explored.

In the present study, spatial excitation patterns were measured in CI patients as functions of electrode configuration, electrode location and stimulation level, using a forward masking paradigm. The distance between electrodes in a bi-polar pair was varied to examine the effect of electrode configuration on the forward masking patterns. The location of stimulation was varied to explore any local effects of the various electrode configurations. The stimulation level was varied to see whether the shape of excitation patterns changed according to loudness.

\section{MATERIALS AND METHODS}

\section{Subjects}

Three adult, post-lingually deafened CI patients participated in the present study; all were users of the Nucleus-22 CI (SPEAK strategy). All three were considered to be highly trained subjects, as they had participated in many previous psychophysical studies in our laboratory. Table 1 lists relevant subject information.

\section{Stimuli}

The Nucleus-22 CI device has 22 electrodes in the array, numbered 1 through 22 from the basal to the 
TABLE 1

\begin{tabular}{|c|c|c|c|c|c|}
\hline \multicolumn{6}{|c|}{ Relevant information for the three $\mathrm{Cl}$ subjects who participated in the experiment } \\
\hline Subject & Age & Etiology & Cl experience (years) & Vowel recognition (\% correct) & Consonant recognition (\% correct) \\
\hline S1 & 57 & Unknown & 8 & 73 & 72 \\
\hline S2 & 41 & Trauma & 6 & 92 & 81 \\
\hline S3 & 60 & Hereditary & 7 & 78 & 75 \\
\hline
\end{tabular}

Phoneme recognition was measured for 12 vowels (10 talkers) and 20 consonants (10 talkers).

apical end of the cochlea. Electrodes are spaced 0.75 $\mathrm{mm}$ apart. The array does not extend fully to the apex of the cochlea, and the insertion depth of the array ultimately depends upon individual patients' cochlear anatomy and the surgical technique. All stimulation is delivered to bi-polar electrode pairs in the Nucleus-22 device as bi-phasic, charge-balanced trains of current pulses. The stimulation mode refers to separation between the active and return electrodes, which determines the extent of the electric field. $\mathrm{BP}$ is the narrowest stimulation mode, in which current flows between adjacent electrodes. $\mathrm{BP}+1$ is a commonly used stimulation mode, in which the active and return electrodes are separated by one electrode (or, $1.5 \mathrm{~mm}$ ). $\mathrm{BP}+21$ is the widest stimulation mode possible in the Nucleus-22 device, in which active and return electrodes are separated by 21 electrodes (or $16.5 \mathrm{~mm}$ ).

In the present study, all stimuli were presented via custom research interface (Shannon et al. 1990). All stimuli were 500 pulses-per-second biphasic pulse trains $(200 \mu \mathrm{s} /$ phase, $20 \mu \mathrm{s}$ inter-phase gap). All probe stimuli were $20 \mathrm{~ms}$ in duration; the stimulation mode was fixed at $\mathrm{BP}+1$. Ten probe electrode pairs were distributed evenly along the electrode array to measure the spread of excitation of a masker stimulus across the entire range of the array. Probe electrode pairs included $(2,4),(4,6),(6,8),(8,10),(10,12)$, $(12,14),(14,16),(16,18),(18,20)$ and $(20,22)$. All masker stimuli were $200 \mathrm{~ms}$. Two sets of masker electrodes were generated. In masker Set A, three masker electrode pairs $[(10,12),(8,14)$, and $(6,16)]$ were selected to vary the extent of electrical stimulation relative to a fixed central location. Thus in masker Set $\mathrm{A}$, the stimulation mode was varied from $\mathrm{BP}+1$, to $\mathrm{BP}+5$, to $\mathrm{BP}+9$, corresponding to $1.5,4.5$ and $7.5 \mathrm{~mm}$ between the active and return electrodes, respectively. In masker Set B, three masker electrode pairs $[(18,20)$, $(10,20)$, and $(2,20)]$ were selected to vary the extent of electrical stimulation relative to a fixed apical location. Thus in masker Set B, the stimulation mode was varied from $\mathrm{BP}+1$, to $\mathrm{BP}+9$, to $\mathrm{BP}+17$, corresponding to $1.5,7.5$ and $12.75 \mathrm{~mm}$ between the active and return electrodes, respectively. Figure 1 shows a schematic diagram of the experimental masker electrode pairs.
Forward-masked thresholds were measured for a variety of masker stimulation levels. For maskers and probes, accurate estimates of current delivered were obtained from the manufacturer's amplitude calibration tables for each subject's device. In the Nucleus22 device, current is delivered to the implant in amplitude steps that are approximately logarithmically spaced. One amplitude step (or, 'clinical unit') corresponds to an increment of approximately $0.2 \mathrm{~dB}$.

The inter-stimulus delay between masker offset and probe onset was fixed at $5 \mathrm{~ms}$. This short inter-stimulus delay allowed for only short-term recovery from masking, which may be associated with more peripheral processes (Brown et al. 1996; Chatterjee 1999). The 5ms masker-probe delay allowed the spatial excitation patterns to be measured at the periphery, and presumably would reflect the neural excitation provided by electrical stimulation.

\section{Procedures}

The dynamic range (DR) for all masker and probe electrode pairs were estimated before the forward masking experiments were begun. Auditory thresholds were measured using a two-alternative forced-

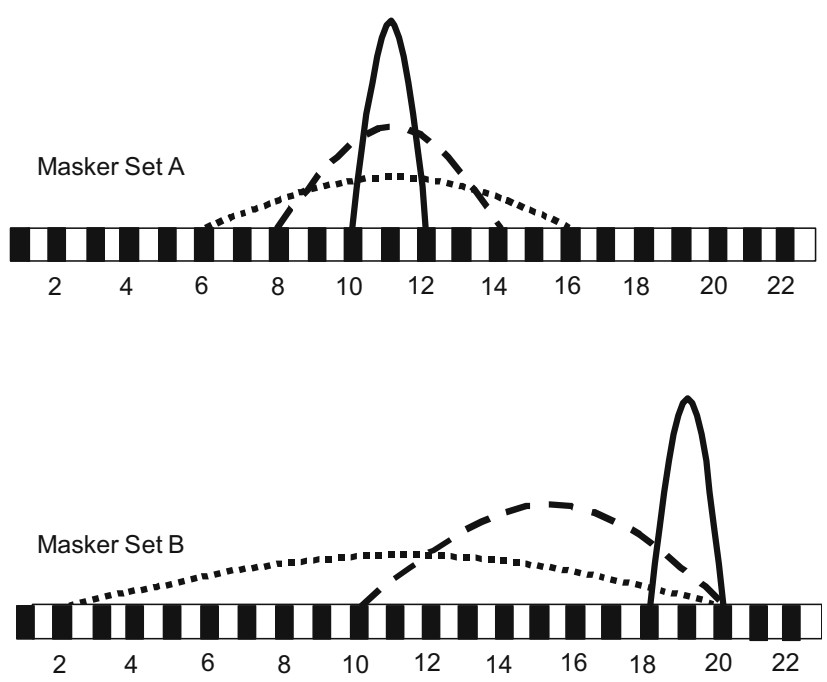

FIG. 1. Experimental electrode configurations for masker set A (top) and set B (bottom). 
choice (2AFC), 3-down/1-up adaptive procedure (visual feedback provided). The amplitude was adjusted according to subject response, converging at $79.4 \%$ correct (Levitt 1971). In each run, there was a maximum of 12 reversals (or 60 trials, which ever came first), and the mean of the last eight reversals was calculated to be the threshold. The average of two runs was calculated to be the threshold for each electrode pair. To obtain the maximum acceptable loudness level (MAL), the subject raised or lowered the amplitude by clicking the left or right buttons of the mouse, respectively, until achieving the MAL. Increments and decrements were set to very small values relative to the stimulus being tested. DR was calculated as the amplitude difference between threshold and MAL. Table 2 shows the threshold, MAL and DR measurements for experimental masker electrode pairs, for all subjects. Note that because subject S2 was unavailable for the complete experiment, it was not possible to measure thresholds, MALs or forwardmasked thresholds for masker electrode pair $(10,20)$.

Forward-masked excitation patterns were measured at soft, medium and loud listening levels, which typically corresponded to $50 \%, 70 \%$ and $80 \%$ of the masker electrode's DR, with a few exceptions. For subject S1, MALs for masker electrode pairs $(10,12)$ and $(6,16)$ increased significantly from earlier DR estimates, such that $80 \%$ DR sounded relatively soft (most likely, MAL has been previously underestimated by subject $\mathrm{S} 1$ ). In this case, the level of the masker was raised to a comfortably loud level, and all successive measurements were made at the new amplitudes; for these electrode pairs with S1, the soft and medium listening levels corresponded to $70 \%$ and $80 \%$ DR, respectively. Conversely for subject S2, MALs for masker electrode pairs $(18,20)$ and $(2,20)$ decreased significantly from earlier DR estimates, such that $80 \%$ DR was too loud (most likely, MAL has been previously overestimated by subject S2). For these masker electrode pairs, the maskers were presented at 20\%, 50\% and $70 \% \mathrm{DR}$, which corresponded (according to the subject) to soft, medium and comfortably loud listening levels.

For the forward-masking experiment, thresholds were measured for each of the 10 probe electrode pairs, for each experimental masker electrode configuration, electrode location and stimulation level. A 3AFC, 3-down/1-up adaptive procedure was used (visual feedback provided). The amplitude was adjusted according to subject response, converging at $79.4 \%$ correct (Levitt 1971). In each run, there was a maximum of 12 reversals (or 60 trials, which ever came first), and the mean of the last eight reversals was calculated to be the threshold. The average of two runs was calculated to be the masked threshold for each probe electrode pair. In the rare event that the standard deviation of the two means exceeded 1 $\mathrm{dB}$, the measurements were repeated.

\section{RESULTS}

Figure 2 shows thresholds in quiet (filled circles) and in the presence of masker Set A at three masker

TABLE 2

\begin{tabular}{|c|c|c|c|c|c|c|c|c|}
\hline \multicolumn{9}{|c|}{ Masker electrode configurations for 3 subjects } \\
\hline Subject & Masker & $\begin{array}{c}\text { Mm between } \\
\text { masker electrodes }\end{array}$ & Threshold $(\mu A)$ & $M A L(\mu A)$ & $\begin{array}{c}\text { Dynamic } \\
\text { range }(\mu A)\end{array}$ & Threshold $(d B)$ & $M A L(d B)$ & $\begin{array}{l}\text { Dynamic } \\
\text { range }(d B)\end{array}$ \\
\hline \multirow[t]{6}{*}{ S1 } & $(10,12)$ & 1.5 & 192 & 1014 & 822 & 45.67 & 60.12 & 14.45 \\
\hline & $(8,14)$ & 4.5 & 122 & 506 & 384 & 41.73 & 54.08 & 12.35 \\
\hline & $(6,16)$ & 7.5 & 71 & 313 & 242 & 37.03 & 49.91 & 12.8 \\
\hline & $(18,20)$ & 1.5 & 152 & 740 & 588 & 43.64 & 57.38 & 13.74 \\
\hline & $(10,20)$ & 7.5 & 67 & 349 & 282 & 36.52 & 50.86 & 14.34 \\
\hline & $(2,20)$ & 13.5 & 66 & 256 & 190 & 36.39 & 48.16 & 11.77 \\
\hline \multirow[t]{6}{*}{ S2 } & $(10,12)$ & 1.5 & 151 & 1000 & 849 & 43.58 & 60.00 & 16.42 \\
\hline & $(8,14)$ & 4.5 & 59 & 435 & 376 & 35.42 & 52.77 & 17.35 \\
\hline & $(6,16)$ & 7.5 & 39 & 337 & 298 & 31.82 & 50.55 & 18.73 \\
\hline & $(18,20)$ & 1.5 & 188 & 1000 & 812 & 45.48 & 60.00 & 14.52 \\
\hline & $(10,20)$ & 7.5 & & & & & & \\
\hline & $(2,20)$ & 13.5 & 34 & 220 & 186 & 30.63 & 46.85 & 16.22 \\
\hline \multirow[t]{6}{*}{ S3 } & $(10,12)$ & 1.5 & 199 & 811 & 612 & 45.98 & 58.18 & 12.20 \\
\hline & $(8,14)$ & 4.5 & 122 & 386 & 264 & 41.73 & 51.73 & 10.00 \\
\hline & $(6,16)$ & 7.5 & 77 & 283 & 206 & 37.73 & 49.04 & 11.31 \\
\hline & $(18,20)$ & 1.5 & 231 & 745 & 514 & 47.27 & 57.44 & 10.17 \\
\hline & $(10,20)$ & 7.5 & 77 & 271 & 194 & 37.73 & 48.66 & 10.93 \\
\hline & $(2,20)$ & 13.5 & 52 & 222 & 170 & 34.32 & 46.93 & 12.61 \\
\hline
\end{tabular}

Thresholds, maximum acceptable loudness (MAL) levels and dynamic ranges are shown in micro-amperes, and in dB (re: $1 \mu \mathrm{A}$ ) for 500 pulses-per-second, $200 \mathrm{~ms}$ pulse trains (200 $\mu$ s/phase, $20 \mu$ s inter-phase gap). 
$(10,12)$
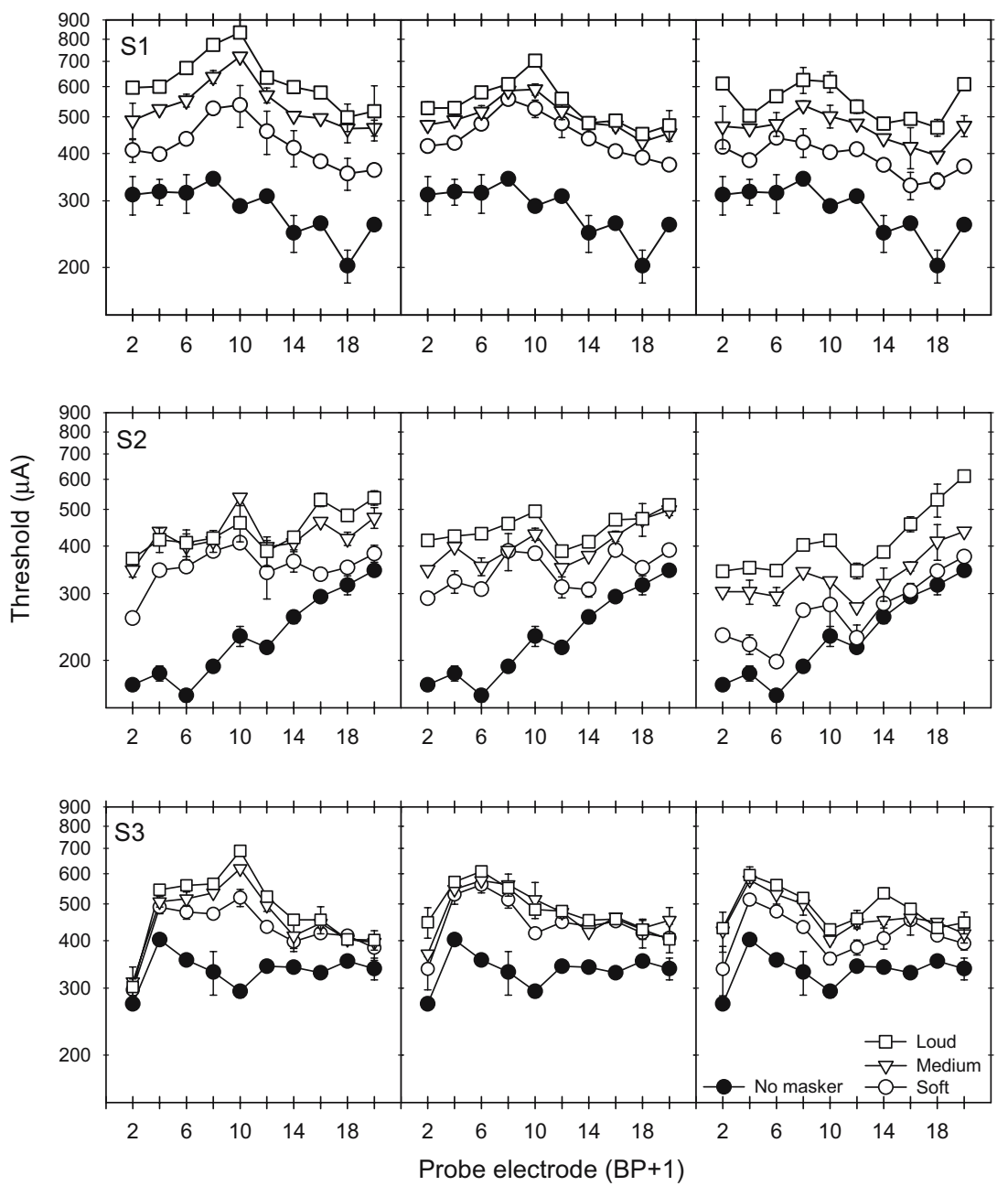

FIG. 2. Masked and unmasked thresholds for the $3 \mathrm{Cl}$ subjects with masker Set A. The filled circles show the absolute detection threshold (no masking) for the probe electrodes. The open symbols show the masked thresholds for maskers presented at soft, medium and loud listening levels.

loudness levels. Each row of panels shows results for individual subjects (S1, S2 and S3, from top to bottom) and each column shows results obtained with a different masker electrode pair $[(10,12),(8,14)$ and $(6,16)$, from left to right]. The $x$-axis shows the probe electrode pair location and the $y$-axis shows the current amplitude in microamperes. For the most narrowly configured masker electrode pair $(10,12)$, masked thresholds were most elevated near the masker location; masked thresholds decayed as the probes became more spatially remote from the masker. For subject S2, masked threshold patterns were less sharply tuned to the masker $(10,12)$, mostly likely because of elevated thresholds in quiet near the apex. As the distance between the active and return electrode in the masker was increased (middle and right columns), the masking patterns became broader. For the most widely configured masker electrode
$(6,16)$, thresholds were most elevated near the masker component electrodes (6 and 16). For all subjects, within each masker electrode pair, as the masker level was increased, the masking pattern simply shifted upward.

Figure 3 shows thresholds in quiet (filled circles) and in the presence of masker Set B at three masker loudness levels. Similar to the masking patterns produced by masker $(10,12)$, masked thresholds were most elevated near masker electrode pair $(18,20)$; masked thresholds decayed as the probes became more spatially remote from the masker. As the distance between the active and return electrode in the masker was increased (middle and right columns), the masking patterns became broader. At the widest masker electrode configuration $(2,20)$, thresholds were most elevated near the masker component electrodes (2 and 20). Again, for all subjects, within 
$(18,20)$

$(10,20)$

$(2,20)$
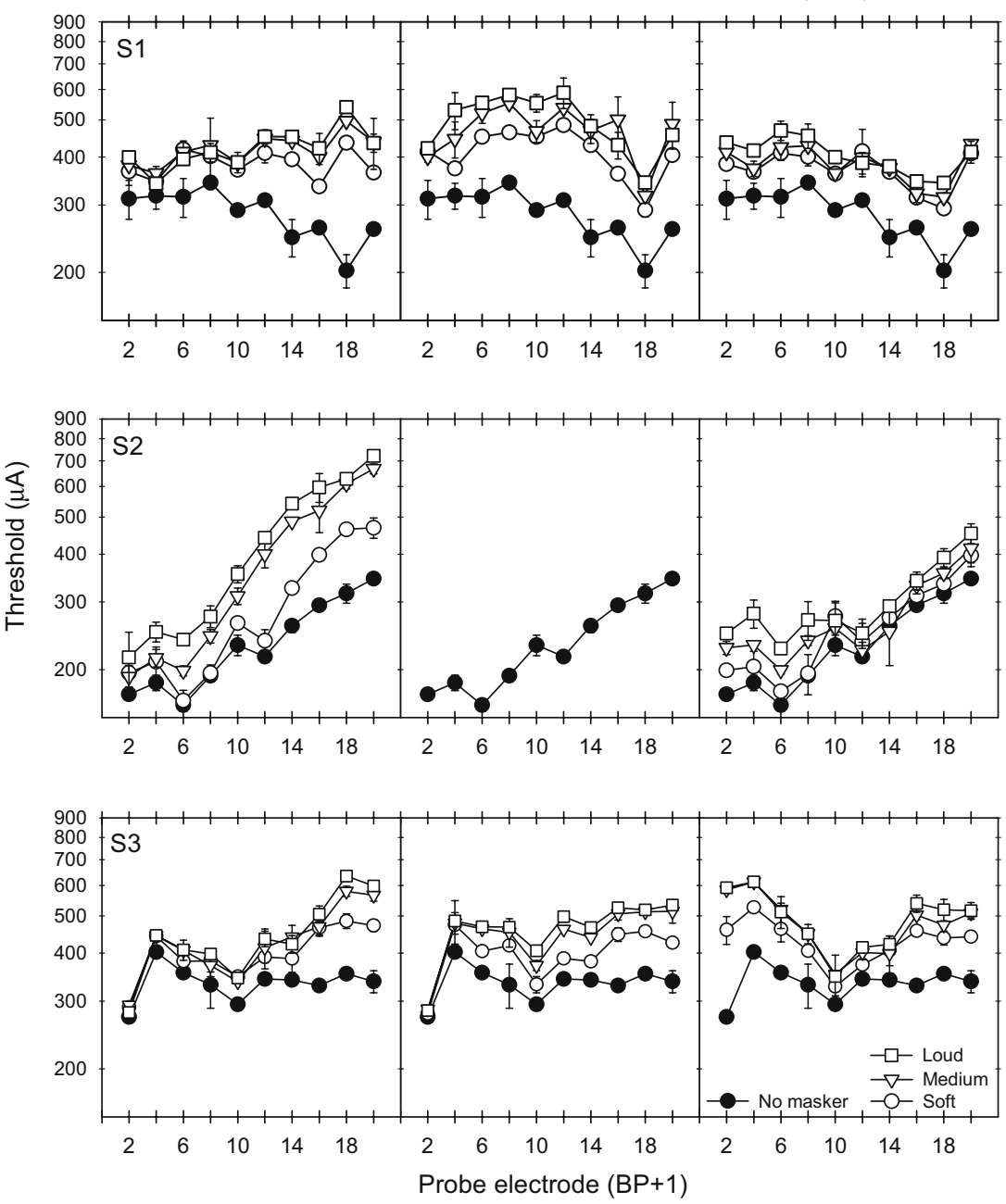

FIG. 3. Masked and unmasked thresholds for the $3 \mathrm{Cl}$ subjects with masker Set B. The filled circles show the absolute detection threshold (no masking) for the probe electrodes. The open symbols show the masked thresholds for maskers presented at soft, medium and loud listening levels.

each masker electrode pair, as the masker level was increased, the masking pattern simply shifted upward.

As seen in Figures 2 and 3, the amount of masking generally increased with masker level and the shape of the masking pattern generally became broader with wider masker electrode configurations. To better compare the relative shape of the excitation pattern for all masker conditions, the forward masking patterns were normalized for each subject and condition. To normalize the masking patterns, the masked threshold shift (in microamperes) at each probe location was divided by the peak masked threshold shift across all probe locations. Figure 4 shows normalized forward masking patterns for masker electrode Set A. The $x$-axis shows the probe electrode pair location and the $y$-axis shows the normalized threshold shift value. The different symbols represent data for different masker levels. Each row of panels shows individual subject data (S1, S2 and S3, from top to bottom) and each column of panels shows data for each masker electrode pair $[(10,12),(8,14)$ and $(6,16)$, from left to right]. As the distance between the active and return electrodes of the masker was increased, the normalized threshold shift patterns changed from a single-peaked, locally distributed pattern (left column) to a broadly spread, sometimes two-peaked pattern (right column). However, as the masker level was increased (different symbols), the normalized masking patterns were largely unchanged. Normalized patterns for masker Set B are shown in Figure 5. Especially for subjects S2 and S3, the shape of masking patterns change from a single-peaked pattern $(18,20)$ to a two-peaked pattern $(2,20)$ as the electrode configuration was made wider.

A three-way ANOVA (treatment factors: probe location, electrode configuration, and stimulation 


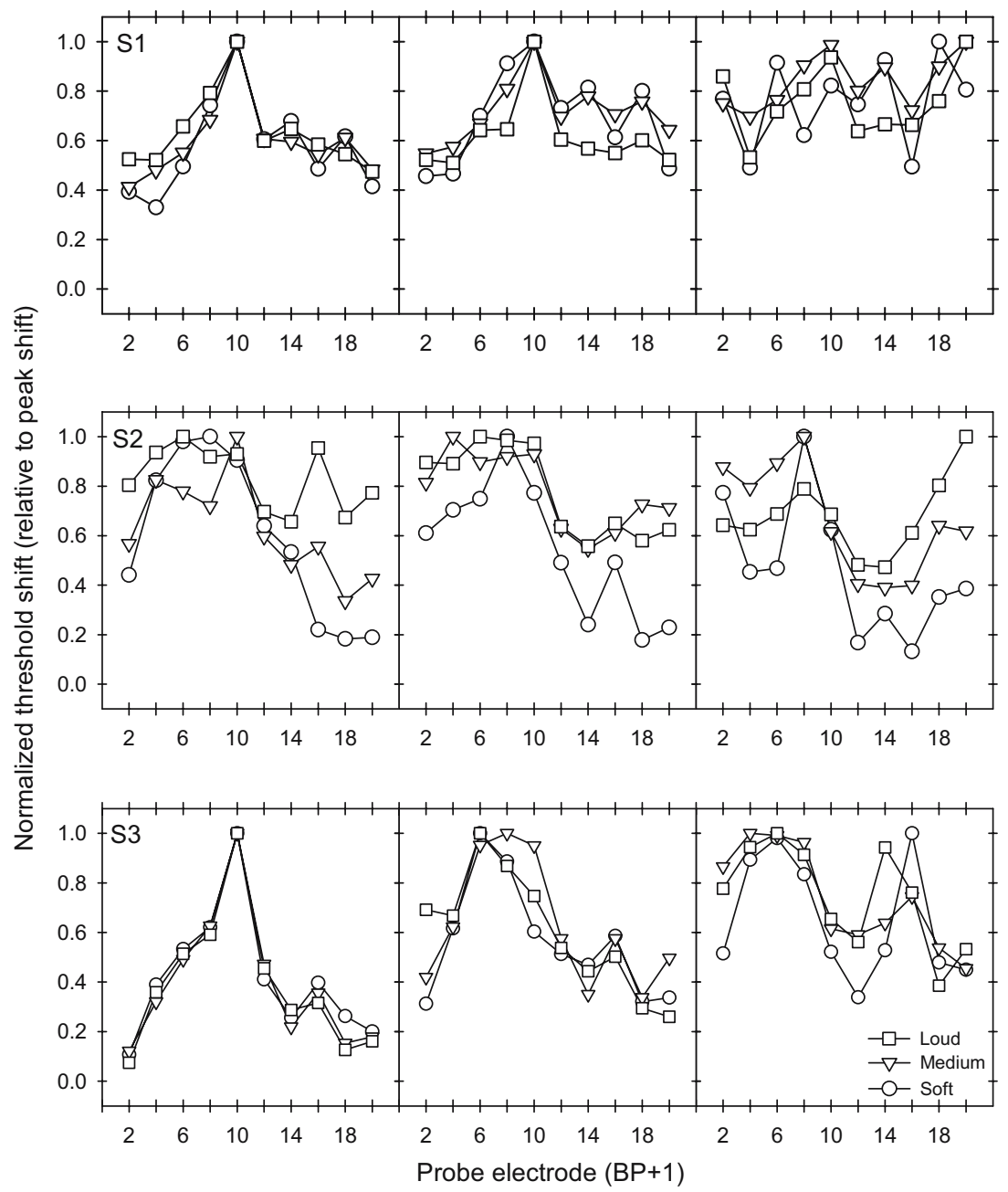

FIG. 4. Normalized threshold shifts for the $3 \mathrm{Cl}$ subjects with masker Set $\mathrm{A}$. Each row of plots shows data for individual $\mathrm{Cl}$ subjects; each column of plots shows data for each masker electrode pair. The open symbols show normalized masked threshold shifts for maskers presented at soft, medium and loud listening levels. In each curve, masked threshold shifts were normalized to the peak shift.

level) was conducted with the normalized data (results shown in Table 3); data were pooled across subjects. Note that for masker Set B, only the narrowest and widest electrode configurations were compared, as there were no data for the medium configuration for subject S2. Statistically significant effects $(p<0.05)$ were found for all factors with one exception: stimulation level did not have a significant effect on the results with masker Set B. No significant interaction was observed between probe location and stimulation level, indicating that the normalized masking patterns did not significantly change as function of stimulation level. However, a significant interaction was observed between probe location and electrode configuration for both masker sets, indicating that the normalized masking patterns significantly changed as function of electrode configuration. No significant interactions were observed between electrode configuration and stimulation level, or between probe location, electrode configuration and stimulation level.

To further compare the relative spread of masking between masker configuration, location and level conditions, the area under the normalized masking pattern was calculated for each experimental condition. Table 4 shows the calculated area for each masker, at each masking level, for each subject. Marked differences were observed between the areas produced by the two masker sets, most likely because of the limited measurable spread with masker Set B. Table 5 shows the results of a two-way ANOVA performed on the data shown in Table 4 (treatment factors: electrode configuration and stimulation level); data was pooled across subjects. Note that for masker Set B, only the narrowest and widest electrode configurations were compared, as there were no data for the medium configuration for subject S2. 
$(18,20)$

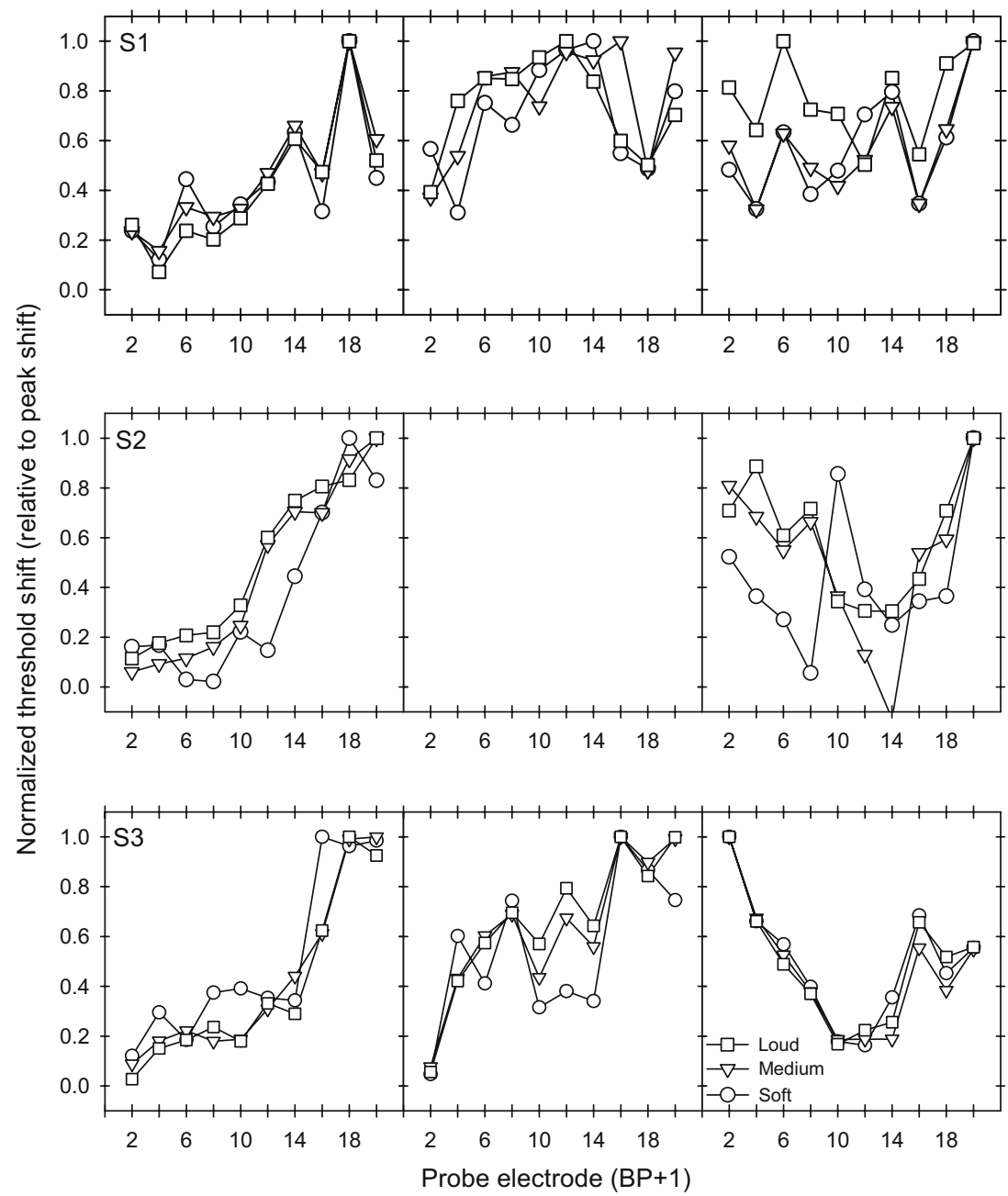

FIG. 5. Normalized threshold shifts for the $3 \mathrm{Cl}$ subjects with masker Set B. Each row of plots shows data for individual $\mathrm{Cl}$ subjects; each column of plots shows data for each masker electrode pair. The open symbols show normalized masked threshold shifts for maskers presented at soft, medium and loud listening levels. In each curve, masked threshold shifts were normalized to the peak shift.

The analyses show that, in most cases, there was no significant difference between the areas under the normalized masking patterns; a significant effect was found with electrode configuration for masker Set B.
Further analysis of the areas under only the singlepeaked masking patterns was also conducted; the results are shown in the bottom three rows in Table 5 (asterisks). No significant difference was found be-

\section{TABLE 3}

Results of three-way ANOVA for normalized masked threshold shift patterns

\begin{tabular}{|c|c|c|c|c|c|c|c|c|}
\hline \multirow[b]{2}{*}{ Treatment factor } & \multicolumn{4}{|c|}{ Masker Set A } & \multicolumn{4}{|c|}{ Masker Set B } \\
\hline & $d F$ & Res & $F$ & $p$ & $d F$ & $\operatorname{Res}$ & $F$ & $p$ \\
\hline Probe location & 9 & 180 & 12.294 & $<\mathbf{0 . 0 0 1}$ & 9 & 120 & 15.436 & $<\mathbf{0 . 0 0 1}$ \\
\hline Electrode configuration & 2 & 180 & 11.492 & $<\mathbf{0 . 0 0 1}$ & 1 & 120 & 15.131 & $<\mathbf{0 . 0 0 1}$ \\
\hline Stimulation level & 2 & 180 & 5.206 & 0.006 & 2 & 120 & 1.588 & 0.209 \\
\hline Probe location $\times$ Electrode configuration & 18 & 180 & 2.089 & 0.008 & 9 & 120 & 11.726 & $<\mathbf{0 . 0 0 1}$ \\
\hline Probe location $\times$ Stimulation level & 18 & 180 & 0.319 & 0.997 & 18 & 120 & 0.254 & 0.999 \\
\hline Electrode configuration $\times$ Stimulation level & 4 & 180 & 1.042 & 0.387 & 2 & 120 & 1.681 & 0.191 \\
\hline Probe location $\times$ Electrode configuration $\times$ Stimulation level & 36 & 180 & 0.178 & 0.999 & 18 & 120 & 0.567 & 0.916 \\
\hline
\end{tabular}

Treatment factors included electrode configuration, stimulation level and probe location. Statistically significant effects are shown in bold type. 


\section{TABLE 4}

Area under normalized threshold shift curves (see Figures 4 and 5) for $3 \mathrm{Cl}$ subjects

\begin{tabular}{|c|c|c|c|c|}
\hline \multirow[b]{2}{*}{ Subject } & \multirow[b]{2}{*}{ Masker } & \multicolumn{3}{|c|}{ Masker level } \\
\hline & & Soft & Medium & Loud \\
\hline \multirow[t]{6}{*}{ S1 } & $(10,12)$ & 10.72 & 11.04 & 11.70 \\
\hline & $(8,14)$ & 13.02 & 13.18 & 11.29 \\
\hline & $(6,16)$ & 13.61 & 15.10 & 13.30 \\
\hline & $(18,20)$ & 7.77 & 8.25 & 7.39 \\
\hline & $(10,20)$ & 12.58 & 14.06 & 13.76 \\
\hline & $(2,20)$ & 10.04 & 9.80 & 13.58 \\
\hline \multirow[t]{6}{*}{ S2 } & $(10,12)$ & 11.20 & 11.58 & 15.11 \\
\hline & $(8,14)$ & 10.09 & 14.04 & 14.07 \\
\hline & $(6,16)$ & 8.12 & 11.76 & 11.95 \\
\hline & $(18,20)$ & 6.46 & 8.07 & 8.95 \\
\hline & $(10,20)$ & & & \\
\hline & $(2,20)$ & 7.32 & 8.60 & 10.32 \\
\hline \multirow[t]{6}{*}{ S3 } & $(10,12)$ & 8.05 & 7.58 & 7.53 \\
\hline & $(8,14)$ & 10.64 & 11.64 & 11.07 \\
\hline & $(6,16)$ & 12.12 & 13.48 & 13.64 \\
\hline & $(18,20)$ & 8.91 & 7.33 & 6.95 \\
\hline & $(10,20)$ & 10.11 & 11.65 & 12.14 \\
\hline & $(2,20)$ & 8.48 & 7.71 & 8.24 \\
\hline
\end{tabular}

tween the areas under these normalized, singlepeaked masking patterns in Masker Set A; a significant difference was found with electrode configuration for Masker Set B. The significant effect of electrode configuration with Masker Set B may have been due to the masker electrode location; near the edge of the electrode array, it is not possible to measure the full extent of the masking pattern, which may have contributed to the difference in the shape of the masking patterns. The statistical analysis of the areas under the normalized masking functions suggests that the spread of masking was quantitatively similar between most experimental conditions despite differences in the shape of the masking pattern.

\section{DISCUSSION}

The results from the present study demonstrate that the pattern of excitation broadens in the cochlea as the distance between the active and return electrode is increased. This result is consistent with previous studies' results, in which forward masking patterns were measured under conditions of varied electrode configurations. For example, Lim et al. (1989) found that, in one CI patient, the masking patterns somewhat broadened as the masker stimulation mode was increased from BP +1 to BP +5 . Shannon (1983) similarly found broader masking patterns for monopolar maskers than for bi-polar maskers. The results presented here show that, for widely separated active and return electrodes located within the cochlea, the excitation pattern spreads broadly across the cochlea; for the widest separations, two peaks in the pattern were often observed. This result is not surprising, given that when component electrodes of an electrode pair are widely separated, the resultant electric field approximates the sum of two independent monopoles. While the widest electrode configurations sometimes seemed to produce less overall masking (as seen in Figures 2 and 3), the relative amount of masking was similar across test conditions (as seen in from the comparable areas under the normalized masking functions in Table 4). This result further supports the notion that electrical fields spread around the component electrodes in a bipolar pair. When the component electrodes are spatially distant, two distinct electrical fields may spread around the active and return electrodes, with a null in the tonotopic space where the fields do not overlap. Recent physiological data obtained in the inferior colliculus of guinea pigs support these findings (Snyder et al. 2005).

Depending upon the location of the extracochlear ground electrode, such an effect may also be present

TABLE 5

\begin{tabular}{|c|c|c|c|c|c|c|c|c|}
\hline \multicolumn{9}{|c|}{ Results of two-way ANOVA for area under normalized masked threshold shift patterns } \\
\hline \multirow[b]{2}{*}{ Treatment factor } & \multicolumn{4}{|c|}{ Masker Set A } & \multicolumn{4}{|c|}{ Masker Set B } \\
\hline & $d F$ & Res & $F$ & $p$ & $d F$ & Res & $F$ & $p$ \\
\hline Electrode configuration & 2 & 18 & 2.369 & 0.122 & 1 & 12 & 5.002 & 0.045 \\
\hline Stimulation level & 2 & 18 & 1.187 & 0.328 & 2 & 12 & 0.951 & 0.414 \\
\hline Probe location $\times$ Electrode configuration & 4 & 18 & 0.309 & 0.868 & 2 & 12 & 1.01 & 0.393 \\
\hline Electrode configuration* & 1 & 6 & 4.549 & 0.077 & 1 & 6 & 45.404 & $<\mathbf{0 . 0 0 1}$ \\
\hline Stimulation level* & 2 & 6 & 0.058 & 0.994 & 2 & 6 & 0.165 & 0.852 \\
\hline Probe location $\times$ Electrode configuration* & 2 & 6 & 0.160 & 0.856 & 2 & 6 & 1.477 & 0.301 \\
\hline
\end{tabular}

The top three rows show comparisons between all available normalized masking patterns in each masker electrode set. The asterisked conditions (bottom three rows) show comparisons between only the single-peaked functions in each masker electrode set for subjects S1 and S3, specifically (10,12) and (8,14) for Masker Set A and $(18,20)$ and $(10,20)$ for Masker Set B. Treatment factors included electrode configuration and stimulation level. Statistically significant effects are shown in bold type. 
in monopolar stimulation; in the Nucleus-24 device, monopolar stimulation is essentially bi-polar, except that the return electrode is located outside the cochlea. In the monopolar case, although current may spread around the active and extra-cochlear return electrodes, there would be no spread of excitation within the cochlea due to the current spread around the return electrode. Consequently, monopolar and narrow bi-polar maskers might produce similar masking patterns within the cochlea, with the greatest amount of masking around the active electrode. Indeed, psychophysical results obtained by Cohen et al. (2001) using an extra-cochlear band in the N-22 device as the monopolar ground, show little difference between masking patterns created by bipolar and monopolar stimuli. Recent psychophysical results (Kwon et al. 2003) comparing forward masking by bi-polar and monopolar stimuli show similar results in Nucleus-24 patients. Although such results appear to be in conflict with observed differences between monopolar and bi-polar stimulation in physiological studies (Bierer and Middlebrooks 2002; Snyder et al. 2004, 2005), it is important to note that differences in current amplitude and the specific locations of the monopolar ground electrode should be be considered when comparing psychophysical and physiological data.

The results of the present study also demonstrate CI patients' sensitivity to location of stimulation when measuring the spread of excitation. Previous forward masking studies with CI patients have similarly shown that the shape of the masking pattern varies considerably across electrode locations within subjects, and across subjects for a fixed electrode location (Shannon 1983; Lim et al. 1989; Throckmorton and Collins 1999; Chatterjee and Shannon 1998). Note that in the present study it was not possible to measure the full extent of masking in the cochlea, because the electrode array is limited in length. As a result, all masking patterns are truncated to some degree; the patterns measured for the widest electrode configurations and/or the most-apical locations were the most truncated.

The present study also demonstrates that stimulation level significantly affected the spread of excitation, and that excitation grew more or less linearly with masker amplitude, consistent with previous studies (Chatterjee and Shannon 1998); increasing the masker level did not change the shape of the masking pattern. This does not mean that the spread of excitation is limited in electrical hearing; as shown in Figures 2 and 3, the amount of masking was clearly greater at higher masker levels. Rather, the normalized masking functions shown in Figures 4 and 5 suggest that the relative spread of excitation was similar at all masker levels. This analysis further supports the idea that the shape of the masking pattern did not change significantly with masker level. The analysis of the area under the normalized masking patterns suggests that while the shape of the masking pattern may have changed with masker electrode configuration and location, the relative spread of masking was similar across masker configuration, location and level.

Interpreting excitation patterns derived from forward masking data presents some difficulties, especially when CI patients' non-uniform neural survival is considered. When a masker or probe is presented to an under-populated (or even 'dead') region, where few or no neurons survive, how should the threshold shift be interpreted? While unmasked thresholds may somewhat reflect the neural health at each location, the masked threshold shift is likely to be related to the responses of a remote or more widely spread group of neurons. The unmasked probe thresholds obtained in the three subjects in this study certainly indicate that we cannot assume uniform nerve survival across the tonotopic axis.

It is reasonable to assume that forward-masking patterns measured here are largely reflective of peripheral excitation patterns, although central contributions cannot be ruled out. It may be argued that the relevance of the precise shape of such patterns to everyday auditory listening is questionable. For example, central processes may only attend to some critical aspect of the spectral profile provided by the periphery, and ignore neural inputs that are not relevant to the listening task. Thus, CI users' speech recognition performance has not been clearly and consistently correlated with spatial selectivity. Indeed, previous studies have not shown any clear advantage for speech recognition with bi-polar or monopolar stimulation (Pfingst et al. 1997), despite presumed differences in the spread of excitation produced by the two stimulation modes. Fu and Shannon (1999) showed compared 4-channel speech recognition when electrodes were configured in bi-polar or pseudo-monopolar stimulation modes. Performance was most similar between the BP + 1 mode [electrodes: $(20,22),(14,16)$, $(8,10),(2,4)]$ and the pseudo-monopolar mode in which the basal electrode was fixed [electrodes: $(2,22)$, $(2,16),(2,10),(2,4)]$; when the apical electrode was fixed [electrodes: $(20,22),(16,22),(10,22),(2,22)]$, performance dropped significantly. Fu and Shannon (1999) therefore suggested that CI listeners might attend more closely to the apical component of a bipolar pair than the basal component. Thus, for the widest electrode configurations tested in the present study (which produced multiple peaks in the masking pattern), only the excitation near the apical electrode may be centrally processed. These previous studies' speech recognition results suggest that the 
spread of excitation may not be a limiting factor in CI speech processing in quiet.

Despite these caveats, it is still important to recognize that channel interaction cannot be ruled out as an important factor in speech recognition, particularly in the presence of background noise (e.g., Friesen et al. 2001; Fu and Nogaki, 2005). Forward masking patterns are a reliable measure of such interactions: it is certainly true that they reflect the ability of the auditory system to detect small changes in the pattern of activity across the tonotopic axis. When this kind of information is particularly sparse, as in the presence of noise, such measures of channel interaction may well turn out to be important predictors of patient performance.

\section{ACKNOWLEDGMENTS}

This study was supported by NIH grants RO1 DC01526, RO1 DC04786, and NIH contract N01 DC92100. We thank Ms. Lendra Friesen for the speech scores obtained by the subjects, and Cochlear Corporation for providing us with the subjects' amplitude tables. We are particularly grateful to the subjects for their support and for their continuing participation in our research over the years. We also thank Ben Bonham for useful discussion.

\section{REFERENCES}

Bierer JA, Middlebrooks JC. Auditory cortical images of cochlearimplant stimuli: dependence on electrode configuration. J. Neurophysiol. 87:478-492, 2002.

Bierer JA, Middlebrooks JC. Cortical responses to cochlear implant stimulation. J. Assoc. Res. Otolaryngol. 5:32-48, 2004.

Boex C, Kos M-I, Pellizone M. Forward masking in different cochlear implant systems. J. Acoust. Soc. Am. 114:2058-2065, 2003.

Brown CJ, AbBas PJ, Borland J, Bertschy MR. Electrically evoked whole nerve action potentials in Ineraid cochlear implant users: responses to different stimulating electrode configurations and comparison to psychophysical responses. J. Speech Hear. Res. 3:453-467, 1996.

Chatterjee M. Temporal mechanisms underlying recovery from forward masking in multi-electrode cochlear implants. J. Acoust. Soc. Am. 105(3):1853-1863, 1999.

Chatterjee M, Shannon RV. Forward masked excitation patterns in multielectrode electrical stimulation. J. Acoust. Soc. Am. 103:2565-2572, 1998.

Cohen LT, Saunders E, Clark GM. Psychophysics of a prototype peri-modiolar cochlear implant electrode array. Hear. Res. 155:63-81, 2001.
Franck KH, Xu L, Pfingst BE. Effects of stimulus level on speech perception with cochlear prostheses. J. Assoc. Res. Otolaryngol. 4(1):49-59, 2003.

Friesen LM, Shannon RV, Baskent D, Wang X. Speech recognition in noise as a function of the number of spectral channels: comparison of acoustic hearing and cochlear implants. J. Acoust. Soc. Am. 110(2):1150-1163, 2001.

Fu Q-J, Nogaki G. Noise susceptibility of cochlear implant users: the role of spectral resolution and smearing. J. Assoc. Res. Otolaryngol. 6(1):19-27, 2005.

Fu Q-J, Shannon RV. Effects of electrode configuration and frequency allocation on vowel recognition with the Nucleus-22 cochlear implant. Ear Hear. 20:332-344, 1999.

Kwon BJ, van den Honert C, Parkinson W, Miller D. Comparison of forward masking pattern for different stimulation modes in cochlear implants. Assoc. Res. Otolayngol. Abs: 199, 2003.

LevitT H. Transformed up-down methods in psychoacoustics. J. Acoust. Soc. Am. Suppl. 1(49):467, 1971.

Lim HH, TONG YC, CLARK GM. Forward masking patterns produced by intracochlear electrical stimulation of one and two electrode pairs in the human cochlea. J. Acoust. Soc. Am. 86:971-980, 1989.

Morris DJ, Pfingst BE. Effects of electrode configuration and stimulus level on rate and level discrimination with cochlear implants. J. Assoc. Res. Otolaryngol. 1:211-223, 2000.

Nelson DA, Donaldson GS. Psychophysical recovery from pulsetrain forward masking in electric hearing. J. Acoust. Soc. Am. 112:2932-2947, 2002.

Pfingst BE, Zwolan TA, Holloway LA. Effects of stimulus configuration on psychophysical operating levels and on speech recognition with cochlear implants. Hear. Res. 112:247-260, 1997.

Pfingst BE, Franck KF, Xu L, Bauer EM, Zwolan TM. Effects of electrode configuration and place of stimulation on speech perception with cochlear prostheses. J. Assoc. Res. Otolaryngol. 2(2):87-103, 2001.

SHANNON RV. Multichannel electrical stimulation of the auditory nerve in man II. Channel interaction. Hear. Res. 12: 1-16, 1983.

Shannon RV, Adams DD, Ferrel RL, Palumbo RL, Grandgenett M. A computer interface for psychophysical and speech research with the Nucleus cochlear implant. J. Acoust. Soc. Am. 87:905907,1990

SNyder RL, Bierer JA, Middlebrooks JC. Topographic spread of inferior colliculus activation in response to acoustic and intracochlear electric stimulation. J. Assoc. Res.Otolaryngol. 5:305322, 2004

Snyder RL, Middlebrooks JC, Hetherington A, Rebscher S, Bonham B. The neurophysiological effects of stimulated auditory prosthesis stimulation: summary of electrode configuration effects using the UCSF guinea pig electrode. Eleventh Quarterly Progress Report, NIH contract N01-DC-02-1006, 2005.

Throckmorton CS, Collins LM. Investigation of the effects of temporal and spatial interactions on speech-recognition skills in cochlear-implant subjects. J. Acoust. Soc. Am. 105:861-873, 1999.

TONG YC, CLARK GM. Loudness summation, masking, and temporal interaction for sensations produced by electric stimulation of two sites in the human cochlea. J. Acoust. Soc. Am. 79:19581966, 1986. 